\section{P2-316 ADULT HEIGHT IN RELATION TO CANCER MORTALITY IN THE EUROPEAN PROSPECTIVE INVESTIGATION INTO CANCER AND NUTRITION (EPIC) COHORT}

doi:10.1136/jech.2011.142976k.49

P Wark, * T Norat, E Riboli, on behalf of the EPIC investigators. Imperial College London, School of Public Health, London, UK

Introduction Adult height is a marker for genetic factors as well as for environmental, hormonal and nutritional factors occurring early in life. Evidence so far suggests that taller people are more likely to be diagnosed and die from cancer than shorter people, which we verify in a large multicentre prospective cohort study.

Methods Within the European Prospective Investigation into Cancer and Nutrition (EPIC), standing height was measured in adults (216280 women and 131544 men) from nine countries between 1991 and 1999. Within the follow-up period that comprised 9.8 years on average, 2716 men and 2692 women died of cancer. HRs of cancer mortality according to height were estimated from Cox proportional hazard models adjusted for smoking status, educational level, alcohol consumption, physical activity, weight and waist circumference.

Results Preliminary analyses showed that cancer mortality rates were higher among taller than among shorter men and women. Among men, a $6 \%$ increase in the hazard rate was observed for every $5 \mathrm{~cm}$ increase in height ( $\mathrm{HR}=1.06,95 \%$ CI 1.03 to 1.10$)$. A very similar increase was seen in women ( $\mathrm{HR}=1.06,95 \% \mathrm{CI} 1.02$ to 1.10$)$. Conclusions These initial findings suggest that factors leading to higher attained adult height or its consequences affect cancer mortality rates in Europeans. Further work will include analyses on cancer incidence and site-specific risks. Our observations do not have direct implications for cancer prevention but could point to underlying mechanisms and thereby trigger further research. The latter may lead to public health interventions on the long term.

\section{P2-317 UTILISING MOBILE HEALTH TO COLLECT EPIDEMIOLOGICAL DATA AND SUPPORT CLINICAL CARE FOR CHRONIC DISEASES; CASE PRESENTATION}

doi:10.1136/jech.2011.142976k.50

J Wawira. ${ }^{*}$ Moi University, Eldoret, Kenya

Introduction Mental healthcare remains a challenge in developing countries due to associated stigma, lack of specialists and facilities; and misdiagnosis of such illnesses. This results in inadequate care of patients with increased morbidity and mortality and high cost of care. The chronic nature of mental illnesses makes the little available care insufficient with loss of follow-up. As a result, it is difficult to specifically determine the epidemiology of mental illnesses worsening delivery of mental care. This abstract decribes a project that utilises mobile health and OpenMRS to develop a community based treatment and data collection tool for mental healthcare.

Methodology A mental health project was developed based on OpenMRS to be used in rural Kenya and Ghana. The mobile component is based on ODK running on android phones and used by community health workers (CHW) to undertake rural data collection and care. The CHW perform daily visits to patients at designated health delivery sites and the psychiatrist reviews data collection to assess clinical decisions made.

Results Using clinical data algorithms to aid diagnosis and epidemiological studies on various mental health illnesses, the ratio of access to mental care was increased. There was reduced dropout rates from the program thus supporting long term care that characterises the chronic nature of mental illnesses.
Conclusion Mobile health and ehealth technologies provide a platform for continued collection of epidemiological data while supporting clinical care by merging clinicians and CHW roles. This is crucial in developing countries with few health workers.

\section{P2-318 LONG-TERM (5-10 YRS) RESULTS OF BALLOON MITRAL COMMISSUROTOMY FOR RHEUMATIC MITRAL STENOSIS}

doi:10.1136/jech.2011.142976k.51

${ }^{1} \mathrm{Y}$ P Devi, ${ }^{2} \mathrm{P}$ N S Haritha, ${ }^{3} \mathrm{D}$ Rajasekhar, ${ }^{3} \mathrm{G}$ Subramanyam, ${ }^{3} \mathrm{P}$ Vanajakshamma, ${ }^{3} \mathrm{~V}$ kumar, ${ }^{3} \mathrm{~N}$ reddy, ${ }^{3} \mathrm{P} \mathrm{K}$ Rao. ${ }^{1}$ Kakatiya University, Warangal, India; ${ }^{2}$ Jawaharlal Nehru Technological University, Hyderabad, India; ${ }^{3}$ SVIMS, Tirupati, India

Introduction Percutaneous mitral commissurotomy (PTMC) has been known as an effective therapeutic modality for moderate to severe mitral stenosis. However, long-term results and factors influencing late outcome after PTMC remain to be elucidated.

Methods Retrospective study of symptomatic severe MS patients who underwent PTMC from 1994 to 1999 in our cardiology unit to evaluate the immediate and long term echocardiographic results of PTMC in 158 patients was conducted. We did transthoracic and transoesophageal echocardio- graphic study of all symptomatic severe mitral stenosis patients. Those patients who underwent PTMC were selected for the study.

Results Males constituted $31 \%$ and Females were $68 \%$ of total patient population. The mean age of patients was $31.09 \pm 9.7$ years. Peak mitral valve gradients increased from $8.9 \mathrm{~mm} \mathrm{Hg} \pm 4.3$ at day one to $14.6 \mathrm{~mm} \mathrm{Hg} \pm 9.8$ and $18.5 \pm 8.2$ at 5 years and 10 years respectively. Mean MVG was $4.7 \pm 2.4 \mathrm{~mm} \mathrm{Hg}$ on day one and $9.18 \pm 5.62$ and $11.8 \pm 6.66$ at 5 and 10 years respectively. All patients in AF were above 40Yrs. Successful PTMC was done in $94.89 \%$. Acute complications were anterior leaflet tear, cardiac tamponade. Mortality immediately after PTMC (1- during MVR), at $10 \mathrm{yrs}$ (3 one case secondary to severe $M R$, one patient stroke related, one patient no cardiac cause). Emergency MVR was done in two patients. Failed PTMC was present in five cases; CMV was done in those cases.

Conclusion Restenosis after 10-year follow-up after successful PTMC, was influenced more by Pre PTMC LA size, mitral valve area, subvalvular fusion than on immediate post PTMC parameters.

\section{P2-319 CORD BLOOD VITAMIN D LEVELS ARE ASSOCIATED WITH EARLY LIFE ATOPIC DERMATITIS}

doi:10.1136/jech.2011.142976k.52

${ }^{1} \mathrm{G}$ Wegienka, ${ }^{*} \mathrm{~S}$ Havstad, ${ }^{2} \mathrm{D}$ Ownby, ${ }^{1} \mathrm{E}$ Zoratti, ${ }^{1} \mathrm{C} \mathrm{C}$ Johnson. ${ }^{1}$ Henry Ford Hospital, Detroit, Michigan, USA; ${ }^{2}$ Medical College of Georgia, Augusta, Augusta, USA

Introduction Vitamin D deficiency (25-hydroxyvitamin D $[25(\mathrm{OH})$ $\mathrm{D}]<50 \mathrm{nmol} / \mathrm{l}$ ) is epidemic in many regions. Recent evidence indicates a possible association between low vitamin $\mathrm{D}$ levels and early life allergic disease.

Methods We examined the relationship between cord blood $25(\mathrm{OH})$ $\mathrm{D}$ and atopic dermatitis $(\mathrm{AD})$ in the first 2 years of life in our birth cohort of children born to a predominantly African American population $(67.7 \%)$ of mothers. Pregnant women living in Detroit, Michigan, USA, and its suburbs were enrolled and their children underwent a standardised physician exam at age 2 years. $\mathrm{AD}$ was evaluated by trained physicians. 
Results Among children of African American mothers, 87/329 (26.4\%) ever had $\mathrm{AD}$ while 19/157 (12\%) children of White mothers ever had $\mathrm{AD}$. Overall, cord blood $25(\mathrm{OH}) \mathrm{D}$ levels were lower in children who ever had $\mathrm{AD}$ (geometric means=GM 30.6 vs $35.6 \mathrm{nmol} / \mathrm{l}$, Wilcoxon Rank Sum=WRS $\mathrm{p}=0.02)$; but the difference was driven by White children (GM 39.7 vs $50.9 \mathrm{nmol} / \mathrm{l}$, WRS $\mathrm{p}=0.036$ ) and not African American children (GM 29.4 vs $29.6 \mathrm{nmol} / \mathrm{l}$, WRS $\mathrm{p}=0.81$ ). The association was also modified by season of birth. Lower $25(\mathrm{OH}) \mathrm{D}$ levels were found in children with $\mathrm{AD}$ born during summer (GM 35.8 vs 45.2 , WRS $\mathrm{p}=0.02$ ), fall (GM 28.1 vs 33.8 , WRS $p=0.036$ ) and winter (GM 30.0 vs 33.7, WRS $\mathrm{p}=0.15)$, but not spring (GM 30.8 vs 31.4 , WRS $p=0.90$ ).

Conclusion Cord blood vitamin $\mathrm{D}$ is associated with $\mathrm{AD}$ at 2 years of age in White but not African American children. The association is also influenced by season of birth.

\section{P2-320 ASTHMA TRENDS IN BRAZILIAN CHILDREN AND ADOLESCENTS: RESULTS FROM THE BRAZILIAN NATIONAL SURVEY (PNAD/IBGE) 1998-2008}

doi:10.1136/jech.2011.142976k.53

F C Wehrmeister,* A M Cascaes, J Martínez-Mesa, A J D Barros, A M B Menezes. Federal University of Pelotas, Pelotas, Rio Grande do Sul, Brazil

Introduction Asthma has a great impact on health services. It affects all ages and it is more prevalent in childhood and adolescence. In South America, including Brazil, there are inconsistencies about the disease trends.

Objective To determine asthma trends from 1998 to 2008, in individuals aged $0-19$ years old in Brazil.

Methods We analysed data of asthma prevalence from the Brazilian National Survey - Pesquisa Nacional por Amostragem de Domicílios (PNAD/IBGE) - of 1998, 2003 and 2008. The analysis was adjusted for the sampling design and included 141, 402; 144, 443; and 134, 032 individuals in 1998, 2003 and 2008 respectively. Prevalence was also estimated by Brazilian macro-regions.

Results The prevalence of asthma among children was $7.71 \%$ in $1998,8.11 \%$ in 2003 and $8.48 \%$ in 2008, with an annual increment of $1 \%$. In the Midwest macro-region there was an annual decline in asthma prevalence in order of $1.09 \%$. In the other macro-regions the prevalence has increased from $0.84 \%$ (South) to $1.52 \%$ (Southeast). Among the group aged 10-19 years the prevalence in 1998 was $4.4 \%$, in 2003 was $4.97 \%$ while in 2008 it was $5.48 \%$. The increase was $2.45 \%$ per year. In the North there was an annual decrease of $0.52 \%$. Other macro-regions showed an increase between $0.88 \%$ (Midwest) and $4.12 \%$ (Northeast).

Conclusions Although asthma is decreasing in developed countries, in Brazil the asthma prevalence in children and adolescents seems to have increased in the period between 1998 and 2008.

\section{P2-321 THE RELATIONSHIP BETWEEN BMI AND WHEEZING IN THE INTERNATIONAL STUDY OF ASTHMA AND ALLERGIES IN CHILDHOOD PHASE 2}

doi:10.1136/jech.2011.142976k.54

${ }^{1} \mathrm{G}$ Weinmayr, ${ }^{1,2} \mathrm{~F}$ Forastiere, ${ }^{1} \mathrm{~A}$ Kleiner, ${ }^{1} \mathrm{G}$ Büchele, ${ }^{1,3} \mathrm{~K}$ Wickens, ${ }^{1,4} \mathrm{D}$ Strachan, ${ }^{1} \mathrm{G}$ Nagel. ${ }^{1}$ Institute of Epidemiology and Medical Biometry, Ulm University, Ulm, Germany; ${ }^{2}$ Dipartimento di Epidemiologia, ASL Roma E, Rome, Italy; ${ }^{3}$ Wellington Asthma Research Group, University of Otago, Wellington, New Zealand; ${ }^{4}$ Division of Population Health Sciences and Education St George's, University of London, London, UK

Introduction An inflammatory link and common genetic factors have been discussed regarding the relation between BMI and asthma. We investigated the relation between obesity and asthma symptoms, and effect modification by atopy and parental allergic disease in Phase 2 of the International Study of Asthma and Allergies in Childhood.

Methods In a population-based cross-sectional study, 10600 children aged 8-12 from 22 centres worldwide were studied with standardised parental questionnaires. Height and weight were measured and age- and sex-specific international BMI cut-off-points were used to define obese children. Atopy was assessed with skin prick testing. Adjusted ORs for each centre were combined with meta-analysis random effects models.

Results Obesity was associated with wheeze in the past year in boys (adjusted OR 1.93 (95\% CI 1.46 to 2.57) and girls 1.51 (1.02 to 2.24). In boys and girls, the effect was the same among atopic and non-atopic children. In boys, the OR was increased in children with reported parental allergic disease compared to those without (2.84 (1.93 to 4.18) and 1.26 (0.8 to 1.98), respectively). Adjustment for parental allergy did not change markedly the association between obesity and wheeze, neither in boys (change from 1.95 to 2.02) nor in girls (1.51 to 1.47).

Conclusions Effect modification but no confounding by parental allergy could indicate that parental allergy does not stand for a common preceding cause of BMI and wheeze (eg, a common genetic pathway), but rather suggests that the influence of BMI may depend on the genetic/family background and also differ between boys and girls.

\section{P2-322 LOW PREVALENCE OF HYPERTENSION IN YEMENITE TYPE 2 DIABETIC PATIENTS}

doi:10.1136/jech.2011.142976k.55

${ }^{1} \mathrm{M}$ B Magnazi, ${ }^{2} \mathrm{~S}$ Weitzman, ${ }^{* 1} \mathrm{~N}$ Reshef, ${ }^{1} \mathrm{~T}$ Zornitzki, ${ }^{1} \mathrm{H}$ Knobler. ${ }^{1}$ Kaplan Medical Center, Rechovot, Israel; ${ }^{2}$ Ben Gurion University of the Negev, Beersheva, Israel

Introduction Previous data suggest that the prevalence of hypertension (HTN) in Yemenite Jews is lower than in the general Israeli population. The aim of the present study was to compare the prevalence of HTN between type 2 diabetic patients of Yemenite $(\mathrm{Y})$ and non-Yemenite (NY) origin.

Methods Cross-sectional study in a Diabetes Clinic. Clinical and lifestyle information was collected including a food frequency questionnaire.

Results Sixty three Y and 120 NY diabetic patients were included in the study. The age and sex distributions were similar in the two groups. Patients in the Y group had lower mean weight and waist circumference $(72.3 \mathrm{~kg}$ vs $85.0 \mathrm{~kg}$ and $95 \mathrm{~cm}$ vs $105 \mathrm{~cm}$, respectively, $\mathrm{p}<0.001)$ and their mean HbA1c level was higher $(7.7 \%$ vs $7.2 \%$, $\mathrm{p}=0.015)$. The prevalence of HTN was significantly lower in the $Y$ compared to the NY group ( $63 \%$ vs $83 \%, p=0.003$ ). Patients in the $Y$ group consumed less antihypertensive medications than those in the NY group (1.6 vs 2.5, $\mathrm{p}=0.002$ ), however blood pressure levels were similar in both groups. In a multivariate logistic regression analysis, NY origin was independently associated with a higher prevalence of HTN (OR 3.0, 95\% CI 1.5 to 6.3, $\mathrm{p}=0.0025$ ). There were no significant differences between the two groups in physical activity, total calories consumed and the DASH score.

Conclusion In this study the prevalence of hypertension in Yemenite was significant lower compared to non-Yemenite diabetic patients. Since no differences were found in lifestyle characteristics it is likely that other mechanisms are involved.

\section{P2-323 WITHDRAWN}

